FIAN/TD-3/06

ITEP/TH-20/06

\title{
M-Theory of Matrix Models
}

\author{
A.Alexandrov ${ }^{\mathrm{a}}$ \\ ITEP, Moscow, Russia \\ A. Mironov ${ }^{\mathrm{b}}$ \\ Lebedev Physics Institute and ITEP, Moscow, Russia
}

\author{
A.Morozov ${ }^{\mathrm{c}}$ \\ ITEP, Moscow, Russia
}

\begin{abstract}
Small $M$-theories unify various models of a given family in the same way as the $M$-theory unifies a variety of superstring models. We consider this idea in application to the family of eigenvalue matrix models: their $M$-theory unifies various branches of Hermitean matrix model (including DijkgraafVafa partition functions) with Kontsevich $\tau$-function. Moreover, the corresponding duality relations look like direct analogues of instanton and meron decompositions, familiar from Yang-Mills theory.
\end{abstract}

\section{Introduction}

String theory ${ }^{1}$ approach to analysis of QFT models involves the following seven steps.

1. When one introduces particular QFT model it is usually defined as a perturbation expansion around a given "classical" background.

2. Perturbation theory is usually defined in terms of correlators that are Gaussian integrals. This leads to two corollaries. The nice one is the Wick theorem and the powerful diagram technique. The bad one is divergence of the perturbation series, which is asymptotic series due to the standard Dyson argument. This is causes by expanding integrands without respect to their asymptotics and, therefore, non-trivial phase/branch structure. ${ }^{2}$

3. The divergent perturbation series can be often presented in the form of a "genus expansion" asymptotic series in a single "Planck-constant" parameter, particular terms of the genus expansion being convergent multiple series in numerous other perturbation parameters (called coupling constants or time-variables).

\footnotetext{
${ }^{\mathrm{a}}$ E-mail: al@itep.ru

${ }^{\mathrm{b}}$ E-mail: mironov@itep.ru; mironov@lpi.ru

${ }^{\mathrm{c}}$ E-mail: morozov@itep.ru

${ }^{1}$ We use this term in the spirit of ref. [1, as a name for the broad modern version of quantum field theory (QFT) with numerous applications, of which various superstring models of sub-Planckian physics are important, but particular examples.

${ }^{2}$ Some more clever non-Gaussian expansions where weights are dictated by dominant non-quadratic terms in action are already in agenda (see [2, 3] for different conceptual approaches to the problem), but are still far from any practical significance.
} 
4. Non-perturbative partition function gets contributions from a variety of "classical" backgrounds, each contribution dressed by its own perturbative expansion.

At this stage we get a representation of non-perturbative partition function - i.e. the only quantity which has an objective physical meaning - as a peculiar triple expansion: over "classical backgrounds", asymptotical quasiclassical (genus) expansion near each background, convergent series in powers of coupling constants for each term of the genus expansion. Actually the boundaries between the three levels of hierarchy are not well defined: changes of integration variables easily mix the levels. Worse than that, given this triple expansion (of which we can usually calculate only the first terms), it is rather difficult to understand the structure of the globally defined non-perturbative partition function (beyond any doubt, it is a nice function, just with sophisticated analytical structure, with numerous branchings and, often, essential singularities, which make perturbation series numerous, divergent and difficult to sew). The next three steps are targeted at curing this situation.

5. Of the three levels the highest - sum over backgrounds - is understood the worst. It is often formulated in the language of "instantons": the idea is that this sum has its own hierarchical structure and infinitely many terms in the sum are somehow expressed through multi-instanton configurations (that probably can be build from the single-instanton ones by acting a few operator generators). A nice realizations of this idea exist at the level of ordinary quantum mechanics see [4, 5] and in 4d QFT in the supersymmetric context (where perturbative contributions artificially cancel) 6 .

6. It is well known, however, that instanton calculus is hardly exhaustive: the proper generators, if any, would rather be made from instanton elementary constituents - merons [7, 8. ${ }^{3}$ Also the role of merons in the distribution of roles between the sum over backgrounds and genus expansions, i.e. between the two upper levels of triple expansion, remains under-investigated (as almost everything in meron physics).

7. Different kinds of expansions - emerging when one starts from different QFT models at step 1 - at non-perturbative level are unified in a global partition function. Since it unifies a priori different models it deserves a special name: nowadays it is often called $M$-theory. Being a true result of functional integration, the global partition function inherits a hidden symmetry [10] (coming from the freedom to change integration variables and providing a non-trivial generalization of the Ward-like symmetries): it belongs to a narrow class of integrable $\tau$-functions [11, satisfying physically-mysterious quadratic relations 10, 12. Various models, arising in different limits in the space of couplings (when one or another background gives dominant contribution), are said to be related by duality: sometime, but not always, duality transformations form a small group (but not obligatory $Z_{2}$ or $S L(2$ ), some dualities can look like trialities etc, generically there is no small group at all). Switching on more and more perturbations, one enlarges $M$-theory, and at the end of the day one can arrive at the all-unifying "theory of everything" (nicknamed String Theory in [1]), where every two QFT models are "dual" in the sense that complicated correlators evaluated in one of them are converted by an appropriate analytical continuation into other complicated correlators in another one. However, one can stop at intermediate stages: by restricting classes of correlators (for example, to those of polynomials of original fields ), one obtains $M$-theories for sufficiently small varieties of QFT models.

The task of the present paper will be a brief introduction into $M$-theory of ordinary (eigenvalue) matrix models [10]. This presentation is based on our recent paper [13, which provided a unified derivation of significant results about relations between a priori different matrix models (Kontsevich model [14, complex-matrix model [15, 16] and Hermitean model [17, 18, with its many phases [19, including the Dijkgraaf-Vafa [20] and other [21] branches), obtained during the last decade [22]-26]

\footnotetext{
${ }^{3}$ For Yang-Mills theory it is a puzzling problem: Yang-Mills instanton can be described as a process of creation and annihilation of a monopole-antimonopole pair, splitting of instanton into two merons is used to separate contributions of these individual quasiparticles and can provide proper variables to describe monopole condensation. Thus, the meron calculus should play the central role in confinement mechanism [7. At the same time there is still no clear room for merons in the ADHM construction [9].
} 
and based, in turn, on the long studies of eigenvalue models, since they were first introduced by F.Dyson in 1962 [17].

The duality relations connect the partition functions of the Hermitean and Kontsevich matrix models. The third important eigenvalue model in the same $M$-theory is less popular but still important complex matrix model with partition function $Z_{C}(t)$. Actually, duality expresses $Z_{C}$ through $Z_{K}$ [13.

Like the Hermitean model, the complex one has many phases/branches, differing by shifts of the time-variables, like (13) and (15). We reserve the notation $Z_{C}(t)$ for the simplest, Gaussian phase (similarly, in the present paper $Z_{K}(\tau)$ denotes the simplest, Kontsevich branch of the GKM partition function; for dualities between different branches of GKM see [34]).

Other models, especially the very important unitary one [27, can probably be also included into this matrix-model $M$-theory. ${ }^{4}$

\section{The WEB of matrix-model dualities}

\subsection{Basic instanton and meron decompositions}

Partition function of the Hermitean matrix model has many branches, some are labeled by two functions $W(\xi)$ and $f(\xi)$ [19, 21]: $Z_{W, f}(t)$. These phases are distinguished by existence of a special t'Hooft's - genus expansion, where genus is indeed the genus of the Feynman-t'Hooft fat-graph diagrams. The leading (spherical) terms of this expansion are known as Dijkgraaf-Vafa partition functions [20]. The best studied is the Gaussian phase, $Z_{G}(t)=Z_{\frac{1}{2} M \xi^{2}, 4 M S}(t)$, associated with $W(\xi)=\frac{1}{2} M \xi^{2}$ and $f(\xi)=4 M S=$ const. Here $S=N g, N$ being the size of the Hermitean matrix. $Z_{G}(t)$ is the Toda-chain $\tau$-function [18, while other $Z_{W, f}(t)$ are $\tau$-functions of less known hierarchies.

For polynomial $W$ and $f, Z_{W, f}$ can be expressed through the Gaussian $Z_{G}(t)$, like the multiinstanton partition function would be expressed through the single-instanton one. If the derivative $W^{\prime}(\xi)$ is a polynomial of degree $n$ in $\xi, W^{\prime}(\xi)=\prod_{i=1}^{n}\left(\xi-\xi_{i}\right)$,

$$
Z_{W, f}(t)=\hat{U}_{W \mid G}\left(t \mid t^{(1)}, \ldots t^{(n)}\right)\left\{\otimes_{i=1}^{n} Z_{G}^{(i)}\left(t^{(i)}\right)\right\}
$$

where time-variables $t^{(i)}$ are obtained from $t$ by shifts, so that they describe expansion around the extremum $\xi_{i}$ of $W(\xi)$, and parameters $M_{i}$ and $N_{i}$ of $Z_{G}^{(i)}$ are made from $W^{\prime \prime}\left(\xi_{i}\right)$ and $f\left(\xi_{i}\right)$, see [24, 25, 26, 19, 21, for the details. Operator $\hat{U}\left(t \mid t^{(1)}, \ldots t^{(n)}\right)$ intertwines between elementary constituents, such intertwiners will appear in all duality relations. All these operators have a very special form, typical for the Weyl-Moyal operators [32, 30] and their Batalin-Vilkovisky generalizations [33]: they are quadratic exponentials of the time-variables $t$ and/or time-derivatives.

However, the decomposition formula (11) is only the beginning. Like Yang-Mills instanton is made from two merons, the Gaussian partition function $Z_{G}(t)$ decomposes into the two Kontsevich $\tau$ functions $Z_{K}(\tau)$ [13]:

$$
Z_{G}(t)=\hat{U}_{G \mid K}\left(t \mid \tau_{ \pm}\right)\left\{Z_{K}\left(\tau_{+}\right) \otimes Z_{K}\left(\tau_{-}\right)\right\}
$$

where the variables $\tau_{ \pm}$can be expressed through $t$ in a variety of ways (for particular $t-\tau_{ \pm}$relations this formula was first suggested in [16] and carefully derived in 22]). Accordingly the meron decomposition exists for the other branches described by the polynomial $W$ of degree $n+1$ :

$$
Z_{W, f}(t)=\hat{U}_{W \mid K}\left(t \mid \tau_{ \pm}^{(1)}, \ldots, \tau_{ \pm}^{(n)}\right)\left\{\otimes_{i=1}^{n}\left(Z_{K}^{(i)}\left(\tau_{+}^{(i)}\right) \otimes Z_{K}^{(i)}\left(\tau_{-}^{(i)}\right)\right\}\right.
$$

\footnotetext{
${ }^{4}$ To avoid a confusion, we remind that the term "M-theory" was introduced in [28] for a unified description of the web of superstring models. Progress with this superstring $M$-theory, revived interest [29] to the old landscape-style views on string theory (e.g. in [30 1) and finally brought more attention to the string theory per se, irrespective of its particular applications - even that great as unification of all fundamental interactions and of all cosmological scenarios. The $M$ theory of matrix models, considered in the present paper, has no direct relation to the superstring $M$-theory (which also uses the matrix-models technique [31]). Though mathematically and conceptually similar (and this is what makes our study important), they concern absolutely different physical theories, the very small one, even with no time-evolution (but allowing a detailed and rigorous description), and enormously big one, big enough to include all our world along with entire variety of all other worlds, thinkable and unthinkable.
} 
However, in variance with (2), this formula is not fully investigated in [13], operator $\hat{U}_{W \mid K}$ is not yet explicitly constructed and the relation

$$
\hat{U}_{W \mid G} \otimes_{i=1}^{n} \hat{U}_{G \mid K}^{(i)}=\hat{U}_{W \mid K}
$$

also requires direct derivation. Entire theory of intertwining Weyl-Moyal operators and underlying *-structures [13] remains to be built.

\subsection{Polynomial representations (moment variables)}

$Z_{G}(t)$ and $Z_{K}(\tau)$ are themselves invariant under some restricted changes of variables [13]:

$$
Z_{G}(t)=U_{G \mid G}(t \mid \tilde{t}) Z_{G}(\tilde{t})
$$

and

$$
Z_{K}(\tau)=U_{K \mid K}(\tau \mid \tilde{\tau}) Z_{K}(\tilde{\tau})
$$

In these formulas intertwiners $U_{G \mid G}$ and $U_{K \mid K}$ do not contain time-derivatives: they are just functions of time-variables and we do not write hats over them. As in all previous cases we do not write the somewhat lengthy formulas for transformations of time variables explicitly (they can be found in ref. [13]). In (5]) and (6) these transformations are linear and depend on two free parameters. However, these few parameters can themselves be made arbitrary functions of times, and the twoparametric families of linear transformations generate infinitely large (still special) families of nonlinear transformations. Moreover, among these non-linear transformations there is one that greatly simplifies $Z_{G}(t)$ and $Z_{K}(\tau)$ : namely, all except the first two terms in genus expansions can be made polynomial in time-variables. We call these special choices $\bar{t}$ and $\bar{\tau}$, and actually

$$
\bar{Z}_{G}(t)=\bar{Z}_{G}(\bar{t})
$$

and

$$
\bar{Z}_{K}(\tau)=\bar{Z}_{K}(\bar{\tau})
$$

In these formulas $\bar{Z}$ is defined in the following way: if $\log Z=\sum_{p \geq 0} g^{2 p-2} F_{p}$ is the genus expansion for $Z$, then $\log \bar{Z}=\sum_{p \geq 2} g^{2 p-2} \bar{F}_{p}$ and, all $\bar{F}_{p}$ with $p \geq 2$ are polynomials of $\bar{t}$ or $\bar{\tau}$ variables (with powers growing as $p$ increases). Since in (5) and (6) intertwiners are functions, and - as a corollary in this case contain only terms with $g^{-2}$ and $g^{0}$ in the exponent, they do not show up in (7) and (8). These formulas are the best possible illustration of statement 3 in the Introduction: contributions of given genus are not just convergent, in appropriate coordinates they are polynomial.

The only word of caution to be made is that while (8) holds for conventional (t'Hooft's) genus expansion, in (7) another, - loop - expansion is implied, see [13. The polynomial coordinates exist the in Gaussian case for the t'Hooft expansion as well [35], associated variables are known as moments, but unlike (7) the corresponding relation

$$
Z_{G}(t)=\hat{U}_{G \mid A C K M}(t \mid \mu) Z_{A C K M}(\mu)
$$

involves a new partition function $Z_{A C K M}(\mu)$ : contributions of genera $p \geq 2$ to $\log Z_{A C K M}(\mu)$ are polynomials in $\mu$, but $Z_{A C K M}$ and $Z_{G}$ are different functions. ${ }^{5}$

\footnotetext{
${ }^{5}$ To avoid possible confusion, we give a trivial example illustrating the difference between (5) and (9):

$$
\sin t=\sin \bar{t}=\frac{1}{2 i}\left(\mu-\mu^{-1}\right)
$$

where $\bar{t}=t+2 \pi$ and $\mu=e^{i t}$. The first equality is a model of (5) with a single function $Z(t)=\sin (t)$, while the second equality relates two different functions, $\sin x$ and $\frac{1}{2 i}\left(x-x^{-1}\right)$.
} 


\section{Group theory approach}

Partition functions of eigenvalue matrix models are best defined as the highest weight vectors in the special Verma modules of Virasoro algebras, i.e. are eigenvectors of their maximal (Borel) subgroups:

$$
\hat{L}_{-}^{I} Z_{I}=0
$$

Here $I=G, K, C$ and Virasoro loop operators are:

$$
\begin{gathered}
\hat{L}_{-}^{G}(z)=\sum_{n=-1}^{\infty} \frac{d z^{2}}{z^{n+2}}\left\{\sum_{k=0}^{\infty} k t_{k}^{\prime} \frac{\partial}{\partial t_{k+n}}+g^{2} \sum_{a+b=n} \frac{\partial^{2}}{\partial t_{a} \partial t_{b}}\right\}, \\
\hat{L}_{-}^{K}(z)=\sum_{n=-1}^{\infty} \frac{d z^{2}}{z^{n+2}}\left\{\sum_{k=0}^{\infty}(k+1 / 2) \tau_{k}^{\prime} \frac{\partial}{\partial \tau_{k+n}}+g^{2} \sum_{a+b=n-1} \frac{\partial^{2}}{\partial \tau_{a} \partial \tau_{b}}\right\}+\frac{1}{16 g^{2}} \tau_{0}^{2} \delta_{n,-1}+\frac{1}{16} \delta_{n, 0}, \\
\hat{L}_{-}^{C}(z)=\sum_{n=0}^{\infty} \frac{d z^{2}}{z^{n+2}}\left\{\sum_{k=0}^{\infty} k t_{2 k}^{\prime} \frac{\partial}{\partial t_{2 k+2 n}}+g^{2} \sum_{a+b=n} \frac{\partial^{2}}{\partial t_{2 a} \partial t_{2 b}}\right\}
\end{gathered}
$$

In these formulas

$$
t_{k}^{\prime}=-\frac{1}{2} M \delta_{k, 2}+t_{k}, \quad \tau_{k}^{\prime}=-\frac{2}{3} M \delta_{k, 1}+\tau_{k}
$$

$Z_{W, f}$ satisfies the equations

$$
\hat{L}_{-}^{W} Z_{W, f}=0
$$

where $\hat{L}_{-}^{W}(z)$ differs from $\hat{L}_{-}^{G}(z)$ only by a shift: for generic $W(\xi)$ it can be written as

$$
\sum_{k} k t_{k}^{\prime} \xi^{k-1}=-W^{\prime}(\xi)+\sum_{k} k t_{k} \xi^{k-1}
$$

The function $f(\xi)$ parameterizes different formal-series solutions to this loop equation, possessing t'Hooft's genus expansion. In the Gaussian case of $W(\xi)=\frac{1}{2} M \xi^{2}$, it reduces to $f(\xi)=4 M S=$ const.

It is expected that similar representations exist for generic non-perturbative partition functions (though even the proper analogues of Virasoro algebras are not yet known in most cases). Thus, the relevant formalism of non-linear realizations of symmetries is of universal importance. The realizations are non-linear because of the shifts of time-variables in the Virasoro equations. To understand the structure and symmetries (dualities) of eigenvectors in non-linear representations, it deserves to begin from the simplest examples, with ordinary operators instead of the loop ones. We return to matrix models in section 5 .

\section{Examples of non-linear realizations of symmetries}

In this section $x, y, z, t, \tau$ will play the role of the time-variables $t$ and $\tau$ of the previous sections.

\section{$4.1 \quad \mathbb{R}^{*} \otimes S O(2)$ group}

Consider the $S O(2)$ invariant functions $Z_{N}$ on the real plane $\mathbb{R}^{2}$ that realize the weight $N$ representation of the group of multiplications by real numbers,

$$
\begin{gathered}
\left(y^{\prime} \frac{\partial}{\partial x}-x^{\prime} \frac{\partial}{\partial y}\right) Z_{N}=0 \\
\left(x^{\prime} \frac{\partial}{\partial x}+y^{\prime} \frac{\partial}{\partial y}\right) Z_{N}=N Z
\end{gathered}
$$


where the shifted variables $x^{\prime}=R \cos \phi=x+r, y^{\prime}=R \sin \phi=y$ parameterize the real plane. Since there is no central extension, the maximal subalgebra in this case can be taken to coincide with the entire algebra.

Now one may use the two different strategies to find $Z_{N}$. First of all, one can just note that the $S O(2)$ invariant functions on the real plane $\mathbb{R}^{2}$ depend only on the radial variable $R$, while the second equation in (15), the homogeneity condition implies that $Z_{N} \sim R^{N}$. The main subtlety here is related to the shift of coordinates $\left(x^{\prime}, y^{\prime}\right) \rightarrow(x, y)$ so that one should re-calculate the linearly realized $S O(2)$ symmetry in coordinates $\left(x^{\prime}, y^{\prime}\right)(\phi \rightarrow \phi+\alpha)$ to its non-linear realization in coordinates with shifted origin $(x, y)$.

Thus the "partition function"

$$
Z_{N}=\text { const } \cdot R^{N} \sim\left(1+\frac{2 x}{r}+\frac{x^{2}+y^{2}}{r^{2}}\right)^{N / 2}
$$

The other possible way of calculating is to construct the solution to (15) as a power series in $x$ and $y$, normalized to start with unity:

$$
Z_{N}(x, y)=\left(1+\frac{2 x}{r}+\frac{x^{2}+y^{2}}{r^{2}}\right)^{N / 2}=1+\frac{N}{r} x+\frac{N(N-1)}{2 r^{2}} x^{2}+\frac{N}{2 r^{2}} y^{2}+\ldots
$$

Coefficients in this series can be recurrently determined from (15) (this is the only way to construct solutions available in matrix models).

To compare these two approaches, one can note that there is the identity:

$$
\forall \alpha \quad Z(x, y)=Z(-r(1-\cos \alpha)+x \cos \alpha+y \sin \alpha,-r \sin \alpha-x \sin \alpha+y \cos \alpha)
$$

Let us check it with for the power series:

$$
\begin{gathered}
1+\frac{N}{r}(-r(1-\cos \alpha)+x \cos \alpha+y \sin \alpha)+\frac{N(N-1)}{2 r^{2}}(-r(1-\cos \alpha)+ \\
+x \cos \alpha+y \sin \alpha)^{2}+\frac{N}{2 r^{2}}(-r \sin \alpha-x \sin \alpha+y \cos \alpha)^{2}+\ldots= \\
=1+\frac{N}{r} x+\frac{N(N-1)}{2 r^{2}} x^{2}+\frac{N}{2 r^{2}} y^{2}+\ldots
\end{gathered}
$$

where both sides should be understood as power series in $x, y$ and $\alpha$. For example, the terms without $x$ and $y$ are

$$
\begin{gathered}
-N(1-\cos \alpha)+\frac{1}{2} N \sin ^{2} \alpha+\frac{1}{2} N(N-1)(1-\cos \alpha)^{2}+\ldots= \\
=\frac{N}{2}\left(\sin ^{2} \alpha-4 \sin ^{2} \frac{\alpha}{2}-4 \sin ^{4} \frac{\alpha}{2}+\ldots\right)+\frac{N^{2}}{2}\left(4 \sin ^{4} \frac{\alpha}{2}+\ldots\right)+\ldots
\end{gathered}
$$

For $\alpha=\frac{\pi}{2}$ one gets

$$
Z_{N}(x, y)=Z_{N}(-r+y,-r-x)
$$

This is trivially true, if one knows that $Z$ is a function of $(r+x)^{2}+y^{2}$, but is a non-trivial identity for formal series, if one does not know their sums explicitly.

\subsection{T-duality}

Much closer to the case of matrix models is the following example ${ }^{6}$ :

$$
\left(t \frac{\partial}{\partial t}+\frac{\partial^{2}}{\partial x^{2}}\right) Z=0
$$

\footnotetext{
${ }^{6}$ A simple one-dimensional example that is very close to the matrix model case is considered in detail in [36].
} 
By a change of variable $t=e^{i \tau}$ it is transformed into the heat/Shrödinger equation (among other things, this $t-\tau$ relation emphasizes "un-naturalness" of expansions in integer powers of $t$, the expansion around the point $t=0$ is not very nice from the point of view of the heat equation):

$$
\left(-i \frac{\partial}{\partial \tau}+\frac{\partial^{2}}{\partial x^{2}}\right) Z=0
$$

with the generic solution

$$
Z(x \mid \tau)=\int e^{i p x} e^{i p^{2} \tau} c(p) d p=\int t^{p^{2}} e^{i p x} c(p) d p
$$

In this 2-variable example it is obvious that (22) defines $Z(x \mid \tau)$ ambiguously, and solutions are parameterized by a function $c(p)$ : a counterpart of $f(\xi)$ in $Z_{W, f}$.

Particular solutions of the heat equation include:

- the heat kernel on a complex line = real plane

$$
Z_{1}(x \mid \tau)=\sqrt{\frac{i \pi}{\tau}} e^{-i x^{2} / 4 \tau}, \quad \text { with } \quad c_{1}(p)=1
$$

- the periodic Jacobi $\theta$-function (actually, this $\theta=\theta_{00}$, but we omit the index 00 in what follows)

$$
Z_{2}(x \mid \tau)=\theta\left(\frac{x}{2 \pi} \mid \frac{\tau}{\pi}\right)=\sum_{n=-\infty}^{\infty} e^{i n^{2} \tau} e^{i n x}, \quad \text { with } c_{2}(p)=\sum_{n=-\infty}^{\infty} \delta(p-n)
$$

- the heat kernel on a torus

$$
Z_{3}(x \mid \tau)=\sqrt{\frac{i \pi}{\tau}} e^{-i x^{2} / 4 \tau} \theta\left(\frac{x}{2 \tau} \mid-\frac{\pi}{\tau}\right)=\sqrt{\frac{i \pi}{\tau}} e^{-i x^{2} / 4 \tau} \sum_{n=-\infty}^{\infty} e^{-i \pi^{2} n^{2} / \tau} e^{i \pi n x / \tau} .
$$

Function $c_{3}(p)$, associated with $Z_{3}$, actually coincides with $c_{2}(p)$ : this is the corollary of $T$-duality, which states that

$$
Z_{2}(x \mid \tau)=Z_{3}(x \mid \tau)
$$

Usually $T$-duality is treated as a transcendental relation, derived, for example, with the help of Poisson re-summation trick. However, one may represent all solutions to the heat equation, including $Z_{1}$, $Z_{2}$ and $Z_{3}$, as linear combinations of Hermite rescaled polynomials $\operatorname{He}_{n}(x \mid \tau) \equiv(-i \tau)^{n / 2} H_{n}\left(\frac{i x}{2 \sqrt{-i \tau}}\right)$ (where we denoted through $H_{n}$ the standard Hermite polynomials)

$$
\begin{gathered}
\mathrm{He}_{0}(x \mid \tau)=1, \quad \mathrm{He}_{1}(x \mid \tau)=i x, \quad \mathrm{He}_{2}(x \mid \tau)=2 i \tau-x^{2}, \quad \mathrm{He}_{3}(x \mid \tau)=-6 x \tau-i x^{3}, \\
\operatorname{He}_{4}(x \mid \tau)=-12 \tau^{2}-12 i x^{2} \tau+x^{4}, \quad \ldots
\end{gathered}
$$

since they solve the heat equation. Then, the relations between different partition functions become involved but elementary algebraic relations.

For instance, in representation (24)

$$
e^{i p^{2} \tau} e^{i p x}=\sum_{n=0}^{\infty} \frac{p^{n}}{n !} \mathrm{He}_{n}(x \mid \tau)
$$

while, in order to get an expansion of the dual representation into the Hermite polynomials, one needs to shift the variable $\tau \rightarrow \tau^{\prime}=T+\tau$ and expand in inverse powers of the background $T$. First, we expand in this way $Z_{1}:{ }^{7}$

$$
\frac{1}{\sqrt{\tau}} e^{-i x^{2} / 4 \tau} \longrightarrow \frac{1}{\sqrt{T}}\left(1+\frac{\tau}{T}\right)^{-1 / 2} \exp \left\{-i\left(\frac{x^{2}}{4}\right) \frac{1}{T}\left(1+\frac{\tau}{T}\right)^{-1}\right\}=\sum_{m \geq 0}^{\infty} \frac{i^{m}}{4^{m} T^{m+1 / 2}} \frac{\operatorname{He}_{2 m}(x \mid \tau)}{m !}
$$

\footnotetext{
${ }^{7}$ This formula is a particular case of the celebrated Möller formula, 37] eq.(10.13.22)]
}

$$
\frac{1}{\sqrt{1-z^{2}}} \exp \left(\frac{2 x y z-\left(x^{2}+y^{2}\right) z^{2}}{1-z^{2}}\right)=\sum_{n=0}^{\infty} \frac{1}{n !}\left(\frac{z}{2}\right)^{n} H_{n}(x) H_{n}(y)
$$


Now for the dual representation, one needs to shift in this formula $x$ by $q$ to obtain ${ }^{8}$

$$
\frac{1}{\sqrt{\tau}} e^{-i x^{2} / 4 \tau} e^{i q x / \tau} e^{-i q^{2} / \tau} \longrightarrow \sum_{m \geq 0}^{\infty} \frac{i^{m}}{4^{m} T^{m+1 / 2}} \frac{\operatorname{He}_{2 m}(x-2 q \mid \tau)}{m !}=\sum_{k, m \geq 0}^{\infty} i^{m-k} \frac{(2 q)^{k}}{T^{m+1 / 2}} \frac{\Gamma\left(m+\frac{1}{2}\right)}{\Gamma\left(\frac{1}{2}\right)} \frac{\operatorname{He}_{2 m-k}(x \mid \tau)}{k !(2 m-k) !}
$$

At the level of the heat equation, the $T$-duality states that

$$
\begin{gathered}
\left(\frac{e^{-i x^{2} / 4 \tau}}{\sqrt{\tau}}\right)^{-1}\left(-i \frac{\partial}{\partial \tau}+\frac{\partial^{2}}{\partial x^{2}}\right) \frac{e^{-i x^{2} / 4 \tau}}{\sqrt{\tau}}=-\left.i \frac{\partial}{\partial \tau}\right|_{x=\text { const }}+\frac{\partial^{2}}{\partial x^{2}}-\frac{i x}{\tau} \frac{\partial}{\partial x}= \\
=\frac{1}{\tau^{2}}\left\{-\left.i \frac{\partial}{\partial(-1 / \tau)}\right|_{x / \tau=\text { const }}+\frac{\partial^{2}}{\partial(x / \tau)^{2}}\right\}
\end{gathered}
$$

Accordingly, for every solution $Z(x \mid \tau)$ of the heat equation, the $T$-dual function

$$
\tilde{Z}(x \mid \tau)=\frac{e^{-i x^{2} / 4 \tau}}{\sqrt{\tau}} Z\left(\frac{x}{\tau} \mid-\frac{1}{\tau}\right)
$$

is also a solution.

\section{Spectral-surface approach}

In the case of the Virasoro constraints only a non-linear realization of the symmetry algebra is known: identification of coordinates, where it is realized linearly, remains a puzzle. It is known as the problem of true variables in string theory, and it remains unresolved at all levels, from quantum gravity and entire string theory to matrix models. Thus dualities, described by eqs.(11)-(9) at our present state of knowledge remain non-trivial - as would be (21) at the level of (19), without (16). Therefore, these dualities should be somehow derived. In ref. 13 we did it with the help of an additional structure: by considering various loop operators $\hat{L}_{-}^{I}$ as different asymptotics of a single global loop operator defined on an auxiliary spectral Riemann surface. The same technique is believed to be adequate for all $M$-theories, one just needs to identify appropriate spectral manifolds...

Definition of the global loop operator actually involves several substructures [13. Three are of universal importance, but we formulate them in matrix-model terms, where the spectral parameter $z$ is a single complex variable:

- the choice of the spectral curve $\Sigma$, where the loop parameter $z$ is taking values $(z$ does not lie just on the complex plane, rather $z \in \Sigma$, and $\Sigma$ can be a non-trivial complex curve, a ramified covering of the bare curve $\Sigma_{0}$, which can be either $C$ or Riemann sphere $\bar{C}$ or complex torus $\left.C^{*}\right)$;

- the choice of the Krichever-Novikov-type [38] algebra, to which the loop operators $\hat{L}(z)$ belong, it is specified by the choice of the structure algebra (the Virasoro algebra in the case of eigenvalue matrix models) and allowed singularities on a bare spectral curve $\Sigma_{0}$ (in the Virasoro case allowed are poles and square-root singularities, for $W_{n}$ algebras ramifications of orders that are divisors of $n$ are allowed), conversion to $\Sigma$ resolves the ramifications so that only poles are allowed in $z \in \Sigma$;

- the choice of a Borel subalgebra $\hat{L}_{-}(z)$, which can have non-trivial eigenvectors, in terms of the spectral manifolds this minus-projection can be represented by an integral operator,

$$
\hat{L}_{-}(z)=\oint_{C} K\left(z, z^{\prime}\right) \hat{L}\left(z^{\prime}\right)
$$

${ }^{8}$ To derive this formula one suffices to note that, for the Hermite polynomials,

$$
\frac{\partial H_{n}(x \mid \tau)}{\partial x}=i n H_{n-1}
$$


Particular operators $\hat{L}_{-}^{I}(z)$ arise from the global $\hat{L}_{-}(z)$ in vicinities of particular singularities, poles are associated with $\hat{L}_{-}^{G}$, quadratic ramifications - with $\hat{L}_{-}^{K}$ and $\hat{L}_{-}^{C}$ (more general GKM $\tau$-functions would arise if the structure algebra was $W_{n}$ with higher order ramification points). Duality relations (11)-(3) arise when the contour $C$ in (36) is decomposed into combinations of contours, surrounding different singularities. Most analytical changes of the spectral variable $z \rightarrow \tilde{z}(z), \partial \tilde{z} / \partial \bar{z}=0$ break the structure of minus-projection, but some - preserve it, and they give rise to identities like (5)-(9).

In the case of eigenvalue matrix models there are two additional substructures [13, which at least simplify the theory, but can also appear to have more general significance. They reflect the existence of free-field formalism on Riemann surfaces, and thus are relevant to non-perturbative partition functions ( $\tau$-functions) associated with $2 d$ conformal models.

- The Virasoro algebra can be embedded into the universal enveloping of the Kac-Moody algebra by the Sugawara construction 39, and this embedding, $\hat{L}(z)=: \hat{J}(z)^{2}$ : can be continued to KricheverNovikov-type algebras (in the matrix-model case, the simplest Kac-Moody algebra $\hat{U}(1)$ is relevant, but everything should work for non-perturbative partition functions, associated with all other Kac-Moody algebras - this is the most straightforward generalization of our construction);

- An additional Seiberg-Witten structure [40, 41, a 1-form $\Omega_{S W}(z)$ with special properties, can be used to construct the kernel

$$
K\left(z, z^{\prime}\right)=\frac{\Omega_{S W}(z)}{\Omega_{S W}\left(z^{\prime}\right)}\left\langle\partial \phi(z) \phi\left(z^{\prime}\right)\right\rangle=\Omega_{S W}(z) k\left(z, z^{\prime}\right)
$$

in (36) from the free-field correlator on the spectral curve; actually this kernel can be used to define a multiplication operation of the Kac-Moody currents

$$
(\hat{J} \star \hat{J})(z)=\oint_{C} k\left(z, z^{\prime}\right): \hat{J}\left(z^{\prime}\right)^{2}:
$$

which plays a big role in "string field theory" [42] and/or in "background-independent" description 43. of the matrix model $M$-theory along the lines of refs. 44, 45].

\section{Acknowledgements}

A.A. is grateful to N.Amburg for useful discussions and V.Poberezhny for the kind hospitality.

This work was partially supported by the Federal Program of the Russian Ministry of Industry, Science and Technology No 40.052.1.1.1112, by the grants RFBR 03-02-17373 (Alexandrov), RFBR 0402-16538a (Mironov), RFBR 04-02-16880 (Morozov), by the Grant of Support for the Scientific Schools 8004.2006.2, NWO project 047.011.2004.026, INTAS project "Current Topics in String Theory" and ANR-05-BLAN-0029-01 project "Geometry and Integrability in Mathematical Physics".

\section{References}

[1] A.Morozov, Sov.Phys.Usp. (UFN) 35 (1992) 671-714.

[2] J.Boháčik, and P.Prešnajder, hep-th/0507129; hep-th/0503235.

[3] V.Dolotin, and A.Morozov, Introduction to Non-Linear Algebra, to appear; hep-th/0501235.

[4] S.Coleman, The use of instantons, Erice lectures, 1977

V.Novikov, M.Shifman, A.Vainshtein, and V.Zakharov, Sov.Phys.Usp. (UFN) 25 (1982) 195.

[5] P.Putrov, Path integral in energy representation in quantum mechanics, hep-th/0605169

[6] N.Nekrasov, Adv.Theor.Math.Phys. 7 (2004) 831-864, arXiv:hep-th/0206161

R.Flume, R.Poghossian, and H.Storch, Mod.Phys.Lett. A17 (2002) 327-340

R.Flume, and R.Poghossian, Int.J.Mod.Phys. A18 (2003) 2541. 
[7] C.Callan, R.Dashen, and D.Gross, Phys.Rev. D17 (1978) 2717.

[8] A.Mironov, A.Morozov, and T.Tomaras, J.Exp.Theor.Phys. 101 (2005) 331-340.

[9] M.Atiyah, V.Drinfeld, N.Hitchin, and Yu.Manin, Phys.Lett. A65 (1978) 185.

[10] A.Morozov, Phys.Usp.(UFN) 37 (1994) 1; hep-th/9502091; hep-th/0502010

A.Mironov, Int.J.Mod.Phys. A9 (1994) 4355; Phys.Part.Nucl. 33 (2002) 537.

[11] A.Gerasimov, S.Khoroshkin, D.Lebedev, A.Mironov, and A.Morozov, Int.J.Mod.Phys. A10 (1995) 2589-2614

A.Mironov, A.Morozov, and L.Vinet, Theor.Math.Phys. 100 (1995) 890-899

S.Kharchev, A.Mironov, and A.Morozov, Theor.Math.Phys. 104 (1995) 129-143

A.Mironov, hep-th/9409190; Theor.Math.Phys. 114 (1998) 127.

[12] A.Morozov, hep-th/9810031

K.Saraikin, hep-th/0604176.

[13] A.Alexandrov, A.Mironov, and A.Morozov, to appear

[14] Kontsevich M.L., Funk.Anal.Prilozh. 25 (1991) v. 2, p. 50 (in Russian)

S.Kharchev, A.Marshakov, A.Mironov, A.Morozov, and A.Zabrodin, Nucl.Phys. B380 (1992) 181-240; Phys.Lett. B275 (1992) 311-314

S.Kharchev, A.Marshakov, A.Mironov, and A.Morozov, Nucl. Phys. B397 (1993) 339; Mod.Phys.Lett. A8 (1993) 1047-1062; Int.J.Mod.Phys. A10 (1995) 2015

P.Di Francesco, C.Itzykson, and J.-B.Zuber, Commun.Math.Phys. 151 (1993) 193-219.

[15] Yu.Makkenko, Pis'ma v ZhETF, 52 (1990) 885-888

J.Amborn, J.Jurkievich, and Yu.Makkenko, Phys.Lett. B251 (1990) 517

T.Morris, Nucl.Phys. B356 (1991) 703-728

A.Anderson, R.C.Meyers, and V.Periwal, Phys.Lett. B254 (1991) 89-93.

[16] Yu.Makeenko, A.Marshakov, A.Mironov, and A.Morozov, Nucl.Phys. B356 (1991) 574.

[17] F.J.Dyson, J.Math.Phys. 3 (1962) 140-156

M.L.Mehta, Random matrices, (2nd ed., Academic Press, New York, 1991)

E.Brézin, C.Itzykson, G.Parisi, and J.-B.Zuber, Commun.Math.Phys. 59 (1978) 35

D.Bessis, Commun.Math.Phys. 69 (1979) 147

D.Bessis, C.Itzykson, and J.B.Zuber, Adv.Appl.Math. 1 (1980) 109

C.Itzykson, and J.-B.Zuber, J.Math.Phys. 21 (1980) 411.

[18] A.Gerasimov, A.Marshakov, A.Mironov, A.Morozov, and A.Orlov, Nucl.Phys. B357 (1991) 565.

[19] A.Alexandrov, A.Mironov, and A.Morozov, Int.J.Mod.Phys. A19 (2004) 4127-4165; Teor.Mat.Fiz. 142 (2005) 419-488; Fortsch.Phys. 53 (2005) 512.

[20] R.Dijkgraaf, and C.Vafa, Nucl.Phys. B644 (2002) 3; Nucl.Phys. B644 (2002) 21; arXiv:hep-th/0208048

L.Chekhov, and A.Mironov, Phys.Lett. B552 (2003) 293

H.Itoyama, and A.Morozov, Nucl.Phys. B657 (2003) 53; Phys.Lett. B555 (2003) 287;

Prog.Theor.Phys. 109 (2003) 433; Int.J.Mod.Phys. A18 (2003) 5889

L.Chekhov, A.Marshakov, A.Mironov, and D.Vasiliev, Phys.Lett. B562 (2003) 323; Proc. Steklov Inst.Math. 251 (2005) 254.

[21] A.Alexandrov, A.Mironov, and A.Morozov, Int.J.Mod.Phys. (to appear), hep-th/0412099.

[22] L.Chekhov, hep-th/9509001. 
[23] I.K.Kostov, hep-th/9907060

[24] G.Bonnet, F.David, and B.Eynard, J.Phys. A33 (2000) 6739.

[25] A.Klemm, M.Mariño, and S.Theisen, JHEP 0303 (2003) 051.

[26] A.Givental, Semisimple Frobenius structures at higher genus, math.AG/0008067.

[27] V.Periwal, and D.Shevitz, Phys. Rev. Lett. 64 (1990) 1326; Nucl. Phys. B344 (1990) 731

M.Bowick, A.Morozov, and D.Shewitz, Nucl.Phys., B354 (1991) 496-530

S.Kharchev, and A.Mironov, Int.J.Mod.Phys. A7 (1992) 4803-4824

A.Mironov, A.Morozov, and G.Semenoff, Int.J.Mod.Phys., A10 (1995) 2015.

[28] P.Horava, and E.Witten, Nucl.Phys. B460 (1996) 506-524.

[29] M.R.Douglas, JHEP 0305 (2003) 046.

[30] A.Gerasimov, D.Lebedev, and A.Morozov, Int.J.Mod.Phys. A6 (1991) 977-988.

[31] T.Banks, W.Fischler, S.H.Shenker, and L.Susskind, Phys.Rev. D55 (1997) 5112-5128.

[32] H.Weyl, Z.Phys. 46 (1927) 1; The Theory of Groups and Quantum Mechanics, Dover Publications, New York Inc. (1931)

J.E.Moyal, Proc. Cambridge Phil.Soc. 45 (1949) 99.

[33] I.A.Batalin, and G.A.Vilkovisky, Phys.Lett. B102 (1981) 27; Phys.Rev. D28 (1983) 2567 [Erratum-ibid. D30 (1984) 508]

I.A.Batalin, and E.S.Fradkin, Phys.Lett. B122 (1983) 157

B.L.Voronov, and I.V.Tyutin, Theor.Math.Phys. 50 (1982) 218

A. Schwarz, Commun.Math.Phys. 155 (1993) 249; ibid. 158 (1993) 373

A.Losev, and A.Gorodencev, Dombay Seminars on Berkovits Theory, Dombay, 2003

A.Losev and D.Krotov, hep-th/0603201.

[34] S.Kharchev, and A.Marshakov, Int.J.Mod.Phys. A10 (1995) 1219-1236

A.Mironov, On GKM description of multi-criticality in 2d gravity, Preprint FIAN/TD/16-92.

[35] J.Ambjorn, L.Chekhov, and Yu.Makeenko, Phys.Lett. B282 (1992) 341-348

J.Ambjorn, L.Chekhov, C.F.Kristjansen, and Yu.Makeenko, Nucl.Phys. B404 (1993) 127-172;

Erratum-ibid. B449 (1995) 681.

[36] A.Mironov, Theor.Math.Phys. 146 (2005) 63.

[37] H.Bateman, Higher Transcendental Functions, Volume II, Mc Graw-Hill, 1953.

[38] I.M.Krichever, and S.P.Novikov, Funct.Anal.Appl. 21 (1987) 126; Funct.Anal.Appl. 21 (1987) 294-307 (1987); Funct.Anal.Appl. 23 (1989) 19-33

R.Dick, DESY-89-160; Lett.Math.Phys. 18 (1989) 255

M.Schlichenmaier, Lett.Math.Phys. 19 (1990) 151; Lett.Math.Phys. 19 (1990) 327;

Lett.Math.Phys. 26 (1992) 23, hep-th/9207088

A.Beilinson, and V.Schechtman, Commun.Math.Phys. 118 (1988) 651-701

A.Morozov, Phys.Lett. B196 (1987) 325.

[39] V.G.Knizhnik, and A.B.Zamolodchikov, Nucl.Phys. B247 (1984) 83-103.

[40] N.Seiberg, and E.Witten, Nucl.Phys. B426 (1994) 19. 
[41] A.Gorsky, I.Krichever, A.Marshakov, A.Mironov, and A.Morozov, Phys.Lett. B355 (1995) 466477

A.Marshakov, Seiberg-Witten Theory and Integrable Systems, (World Scientific, Singapore, 1999) H.W.Braden and I.Krichever (Eds.), Integrability: The Seiberg-Witten and Whitham Equations, (Gordon and Breach, 2000)

A.Gorsky and A.Mironov, Integrable Many-Body Systems and Gauge Theories, hep-th/0011197.

[42] E.Witten, Nucl.Phys. B268 (1986) 253

B.Zwiebach, Phys.Lett. B256 (1991) 22-29; Mod.Phys.Lett. A7 (1992) 1079-1090; Annals Phys. 267 (1998) 193-248; hep-th/9305026.

[43] E.Witten, B276 (1986) 291; hep-th/9306122

A.Sen, and B.Zwiebach, Nucl.Phys. B414 (1994) 649-714; B423 (1994) 580-630; Commun.Math.Phys. 177 (1996) 305-326

A.Gerasimov, and S.Shatashvili, JHEP 0106 (2001) 066.

[44] B.Eynard, JHEP 0411 (2004) 031

L.Chekhov, and B.Eynard, hep-th/0504116.

[45] A.Alexandrov, A.Mironov, and A.Morozov, unpublished 